$\xi_{p-1}$

\title{
Design and analysis of proof mass based micro sensor for early detection of Parkinson's disease
}

\author{
G.R.K Prasad*, Syed Shameem, N. Srinivasulu, C. Renukavalli, B. Manasa, P. S. Srinivas Babu \\ Micro Electronics Research Group, Department of ECE, Koneru Lakshmaiah Education Foundation, \\ Vaddeswaram, Guntur, Andhra Pradesh, India-522502 \\ *Corresponding author E-mail: ramguda1978@gmail.com
}

\begin{abstract}
Micro Electro Mechanical System (MEMS) is the promising technology in bio-medical engineering which reduces the cost, sensitivity and accuracy of the devices. A Novel structure is proposed using MEMS technology in this paper. The structure comprises of a proof mass which is movable and four arms or limbs with a torus of $90^{\circ}$ revolution angle. The four edges of the torus are fixed. A fixed plate is kept on the top of the proof mass to measure the capacitance and output voltage of the proposed MEMS sensor. The sensor is designed for a value of $0.4 \mathrm{~g}$ to $0.8 \mathrm{~g}$. The simulated values are correlated with the theoretical values. The proposed design accurately measures tremor frequency which is in the range of $4 \mathrm{~Hz}$ to $7 \mathrm{~Hz}$. The proof mass in the structure moves up and down when the device is attached to finger tip of the patient because the resting tremor or pill rolling tremor is the preliminary and basic detection symptom of Parkinson's disease. When the structure is connected to fingertip, variation in Eigen Frequency, output voltage and capacitance is measured. An amplifier is designed with a gain of $103 \mathrm{~dB}$ and verified with the voltage so obtained from the output of the proposed sensor is 100 milli volts. There is a variation of 5\% change in capacitance from theoretical to simulated values. Similarly, $8 \%$ variation in voltage. The displacement sensitivity is increased by $10 \%$. The design is simulated using FEM tool COMSOL Multiphysics.
\end{abstract}

Keywords: MEMS, Parkinson's Disease, frequency, tremor,Eigen Frequency,Amplifier.

\section{Introduction}

A major technological branch of MEMS is Bio-MEMS or micro electromechanical systems for biomedical applications leads to developmental aspects and research in miniature devices like MEMS by the fusion of traditional MEMS devices with integration of bio medical devices. With the development of such types of MEMS devices [1][2][3], it is easy to predict and analyze some of the parameters and inhibiting characteristics of the diseases. The typical feature's dimension of Bio-MEMS ranges from submicron to micron range $(100 \mathrm{~nm}-200 \mathrm{~mm})$ and the remaining dimensions up to several millimetres. On the other hand, Bio-MEMS may be the crucial component which plays a key role in a larger device such as medical imaging machine. These devices may operate in vitro or vivo (inside or outside a living system) and have self-generated or external power sources. They may operate either in closed loop (auto regulated) or open ended (Sensor - actuator) system.

The damage or long term degenerative disorder of the central nerves system is the main cause the effects the motor symptoms is generally known as Parkinson's Disease. The main symptoms to observe the disease are shaking, rigidity, slowness of movement, and difficulty with walking [4]. The cause of Parkinson's disease is unknown but so many factors are in account like habits, environmental factors etc. The main and observable symptom for Parkinson's disease is shaking of hands which are generally known as resting tremor. The tremor frequency of hands is in the range of $3 \mathrm{~Hz}-8 \mathrm{~Hz}[5][6]$. Early stage detection of Parkinson's disease on of the challenging task.
The micro sensors are the miniature devices with low cost, and easy to handle which in turn will accurately provide the information in the required form to the end user[7][8][9]. Up to now most of the research work was done for early detection of Parkinson's disease with the help of image processing algorithms and speech based tests. We are proposing a novel structure like Oxy meter device made with the help of the MEMS sensors and is fixed to the tip of the finger.

\section{Literature}

Paul Pyzowski et, al used the MEMS based electrode array used in the Brain gate system for quadriplegics to detect the functioning of nerves system which are directly connected to the brain, which causes the Parkinson's disease. They have used the technique called deep brain simulation. They have also applied the same technique cognitive disorders including epilepsy, obsessivecompulsive disorder, and drug resistant depression [10].

PG Gopinath et. al have proposed MEMS based low cost portable devices useful to detect various parameters like sensitivity, potential ability etc[11].

Shyamal patel et.al given review on different wearable sensor systems used in rehabilitation of the patients suffering from the neuro degenerative diseases[12].

Athanasios Tsanas et.al, have estimated PD symptom progress by establishing a mapping between dysphonia measures and UPDRS with help of three non-linear and one non linear regression method[13]. 
Ulrike Sommer et.al, work with transcranial sonography and tested 11 of 30 patients with idiopathic olfactory loss presented themselves with SN hperechogenicity on one or both sides. They have identified 8 patients with clinically detectable symptoms[14].

Ali Saad et.al, identified the presence of Parkinson's disease using a prototype with the help of Freezing of Gait(FoG). The FoG episodes are detected using a multi sensor device for data acquisition and the gaussian neural networks as classification tool. They have incorporated number of sensors in shoe to observe the foot to foot inter distance, and to observe walking disorders[15]. George Rigas et.al proposed an automated method for tremor assessment. They have extracted different parameters from accelerometers mounted in different segments of body, which are used as observations in two running parallel HMM models. The first one is used to quantify tremor severity and the second one is to recognize body posture and action[16].

Zack Zhu et.al have proposed a wearable system that is capable of conducting real time detection of FoG events in PD patients, with the use of the smart phone[17].

Sinziana Mazilu et.al have investigated the correlation between wrist movement and freezing of gait in Parkinsons disease using FoG detection assistant in which sensors are integrated in smart watches and wrist bands like wearable devices[18].

Benoit Mariani et.al have proposed on shoe wearable sensors to instrument commonly used motor function tests for Parkinson's disease. The temporal parameters like stride velocity and stride length are extracted using TUG( Time up and Go) and gait[19]. K.Harish et.al proposed an algorithm to detect the resting tremor of PD patients. For every 3 seconds of time they have extracted the results like frequency of tremor and amplitude of tremor and estimated the severity of tremor[20].

Jens Barth et.al have proposed a sensor based system which is attached to shoe and is able to identify Parkinson's disease associated with gait patterns and to distinguish different levels of gait impairments[21].

\section{Methodology}

Tremor or shaking of hands is the main symptom for early detection of Parkinson's Disease. The vibrational or tremor frequency movement of hands of Parkinson's disease is from $3 \mathrm{~Hz}$ to $8 \mathrm{~Hz}$.

The Eigen frequency of vibrating body is expressed as

$f_{0}=\frac{1}{2 \pi} \sqrt{\frac{K}{m_{\text {eff }}}}$

Where $\mathrm{K}$ is spring constant of the material, $\mathrm{m}_{\mathrm{eff}}$ is the effective mass of the structure.

In the proposed work, we have changed the thickness of the moving proof mass and calculated the effective mass andfrequency of the structure by keeping spacing between the plates constant as shown in figure 1(a) and 1(b). The minimum distance between the plates is $450 \mathrm{um}$.

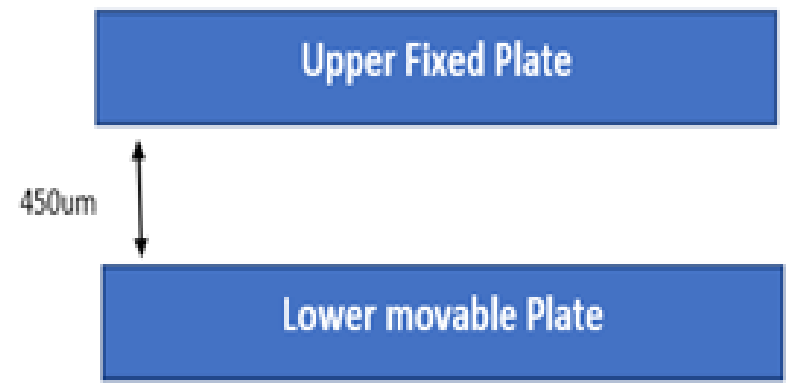

Fig. 1(a): Block Diagram of proposed Structure

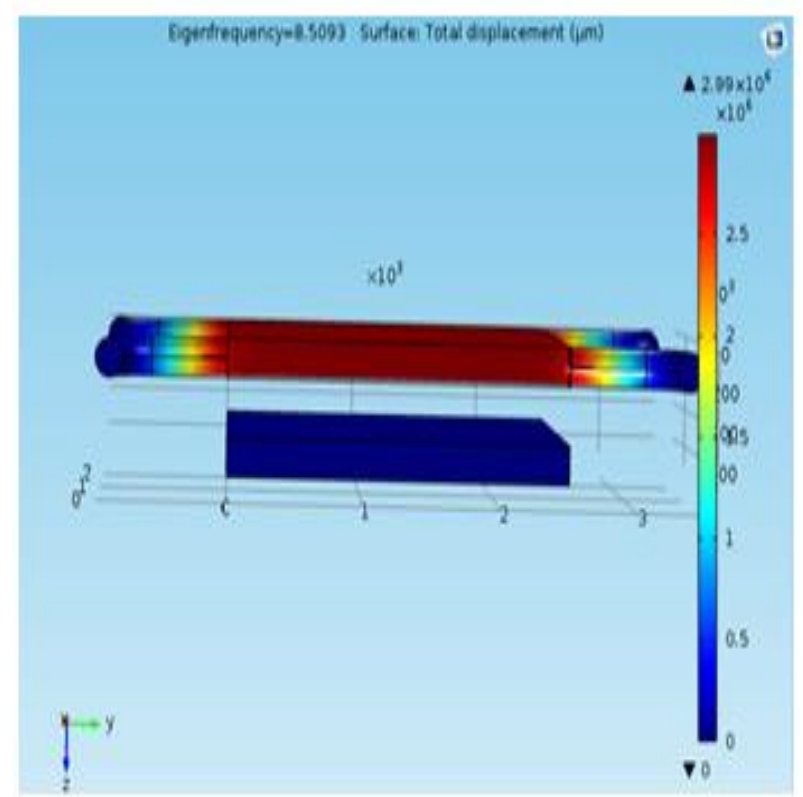

Fig. 1(b): Simulation result of proposed Structure for sensing

In FEM analysis, the meshing structure of the proposed structure is shown in fig 2 and it is simulated in COMSOL Multi Physics FEM tool.

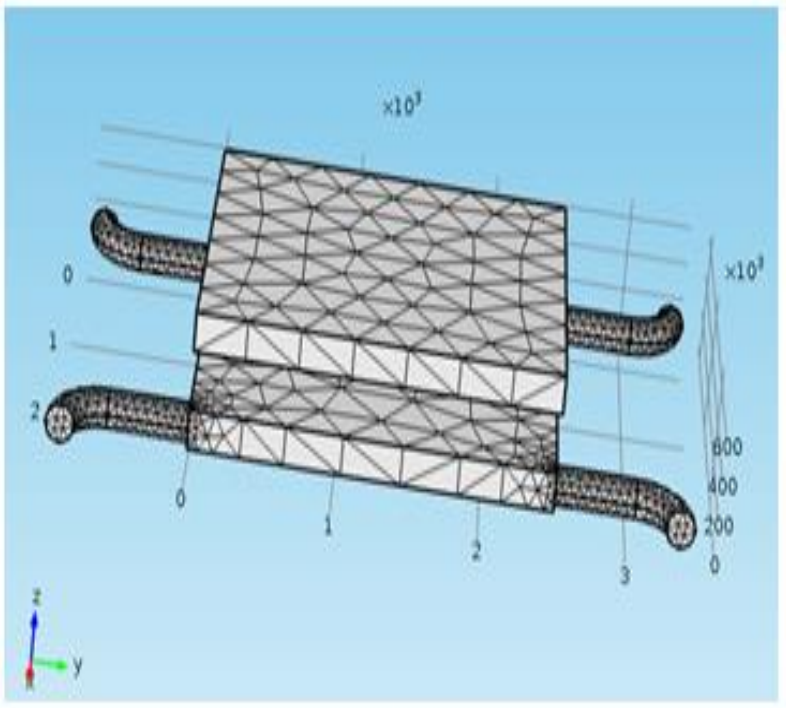

Fig. 2: FEM analysis of sensing element of proposed structure

The block diagram of proposed sensor in total is represented in figure 3

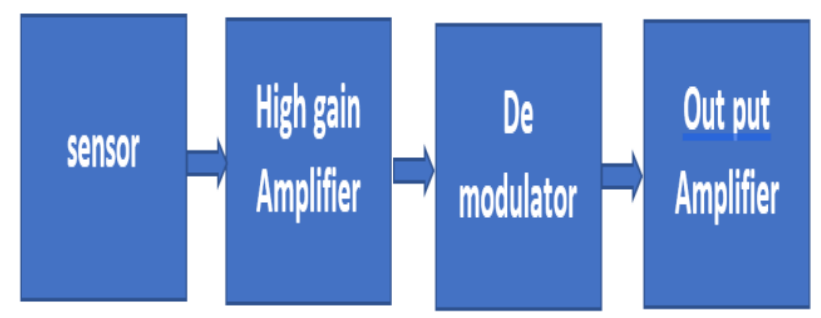

Fig. 3: block diagram of proposed structure

The work flow of the sensor is shown in flow chart in figure 4 


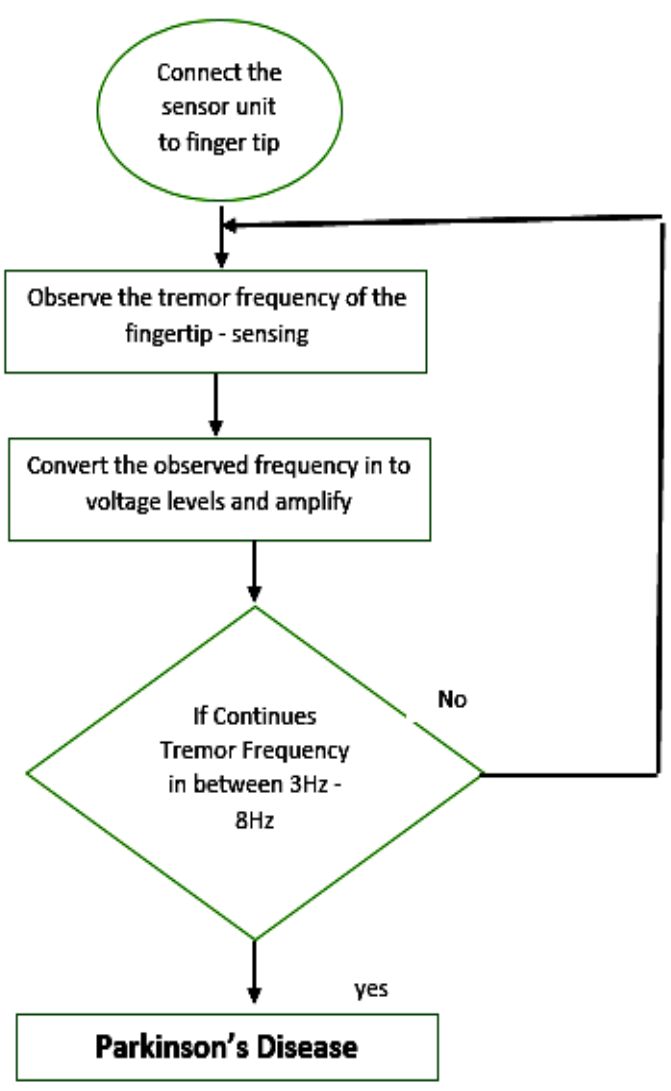

Fig. 4: Flowchart of work flow of proposed sensor

At first the proposed sensor unit is connected to the finger tip of the patient who is suffering from Parkinson's disease. The finger vibration is the input for the sensor connected. Those vibrations are converted with the help of V\Frequency to voltage converter. The obtained voltage is amplifier and again converted in to frequency using voltage to frequency converter and checks whether the frequency is in the range of $3 \mathrm{hz}$ to $8 \mathrm{hz}$. If the continues tremor frequency is in this range, the physician concludes that it is Parkinson's disease.

\section{Materials and Properties}

The Proposed structure was simulated by taking material for proof mass as PTFE and the material for cylindrical limbs and torus are with silicon. The material properties are given in table 1

Table 1: Materials used and its Properties

\begin{tabular}{ccc}
\hline Property/Material & PTFE & Silicon \\
\hline Young's modulus & 410 & 179 \\
\hline Poission'sRatio & 0.46 & 0.26 \\
\hline Density (kg/m3) & 2150 & 2330 \\
\hline
\end{tabular}

\section{Results}

By varying the thickness of the movable proof mass, the eigen frequency and capacitance and voltage between the plates also changes. Here the thickness of the proof mass is varied from 50um to 300um and observed the variation in displacement with respect to variation in eigen frequency.

eigen frequency varies from $4.45 \mathrm{~Hz}$ to $24.3 \mathrm{~Hz}$ as shown in figure $1(\mathrm{~b})$.

The variation in Eigen frequency with the variation in thickness of the proof mass is shown in table 2.
Table 2: Displacement of Proof mass with Eigen Frequency

\begin{tabular}{|c|c|c|c|c|c|c|}
\hline \multirow{2}{*}{$\begin{array}{c}\text { Eigen } \\
\text { frequency } \\
(\mathbf{H z})\end{array}$} & \multicolumn{5}{|c|}{ Displacement of the proof mass (um) } \\
\cline { 2 - 7 } & $\mathbf{5 0}$ & $\mathbf{1 0 0}$ & $\mathbf{1 5 0}$ & $\mathbf{2 0 0}$ & $\mathbf{2 5 0}$ & $\mathbf{3 0 0}$ \\
\hline 4.45 & 2.71 & 2.28 & 2.12 & 2.07 & 2.05 & 2.06 \\
\hline 7.96 & 3.89 & 3.3 & 3.07 & 2.99 & 2.97 & 2.98 \\
\hline 8.49 & 6.79 & 5.23 & 4.87 & 4.74 & 4.71 & 4.72 \\
\hline 11.64 & 6.38 & 5.45 & 5.08 & 4.94 & 4.91 & 4.92 \\
\hline 21.9 & 8.27 & 6.75 & 6.28 & 6.12 & 6.08 & 6.09 \\
\hline 24.3 & 12.1 & 5.19 & 4.83 & 4.71 & 4.68 & 4.68 \\
\hline
\end{tabular}

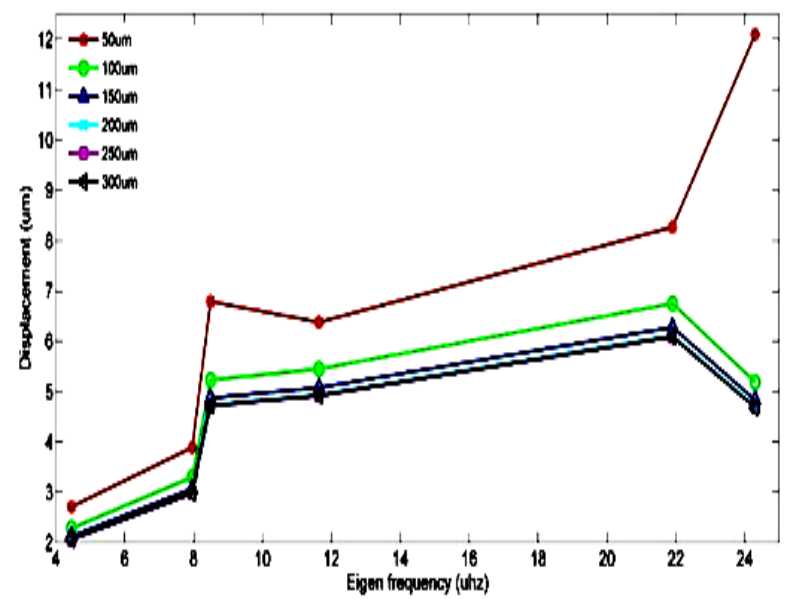

Fig. 5: Variation of displacement with respect to variation in Eigen Frequency for different spacing between plates

From Fig 5 it is observed that for 50 um thickness proof mass the maximum displacement is noted(12.1um) and for $300 \mathrm{um}$ thickness proof mass minimum displacement is noted (4.68um). To get the range of frequency specified for detection of Parkinson's disease, from the calculations the thickness of the proof mass is finalized as $200 \mathrm{um}$.

The sensing methodology is very crucial in microelectronics. As per the studies capacitive sensing gives more and accurate. So we have adopted capacitive sensing for my proposed work. In capacitive sensing, the displacement between plates is taken in to consideration and capacitance is calculated.

The capacitance of a capacitor with respect to change in displacement between the plates is expressed as

$$
C=\frac{\varepsilon_{0}}{d}
$$

where $\mathrm{C}$ is capacitance of the capacitor, $\mathrm{d}$ is spacing between the plates, $\varepsilon_{0}$-permittivity of the medium. The di-electric material between the plates is free space.

The multi slice capacitance variation of the plates is shown in Fig 6 (a) and 6(b) for a plate separation of $500 \mu \mathrm{m}$ and $450 \mu \mathrm{m}$.

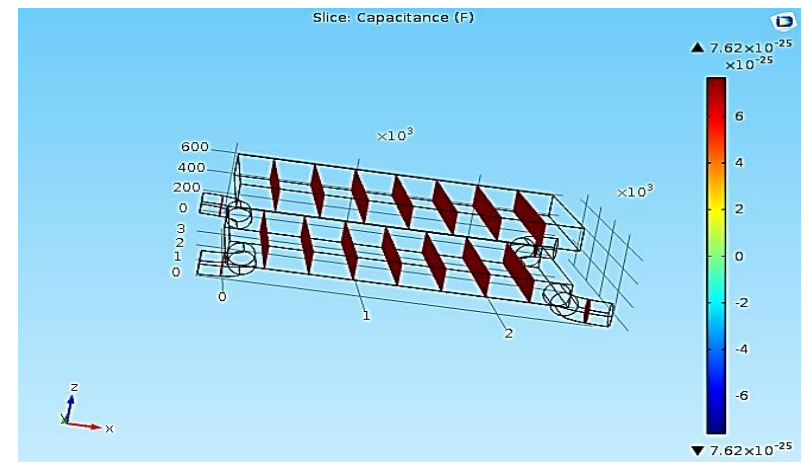

Fig. 6(a): Capacitance when the distance between the plates 500um 


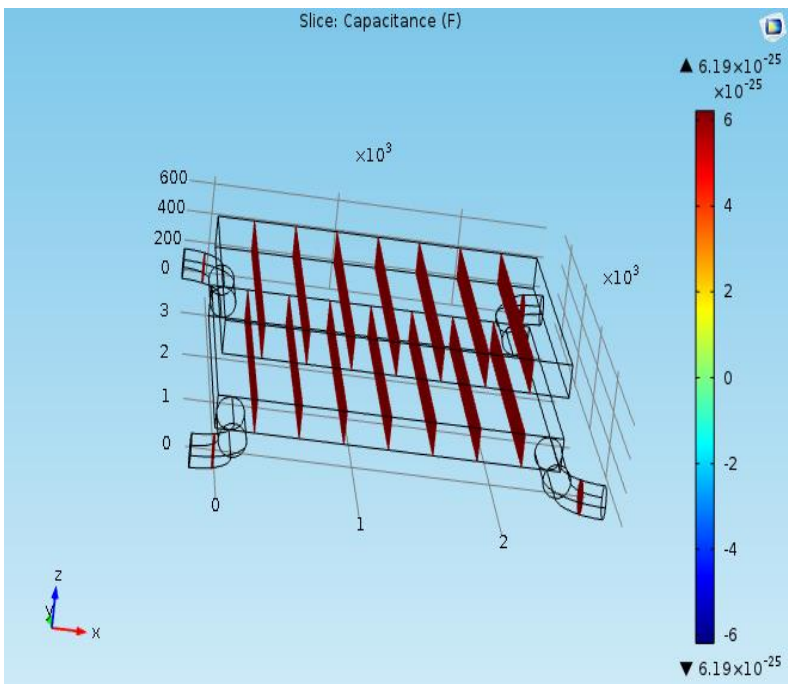

Fig. 6 (b): Capacitance when the distance between the plates 450um

The variation of capacitance with distance between the plates is shown in table 3

Table 3: Capacitance of the proposed model with varying Distance between the plates

\begin{tabular}{|l|l|l|}
\hline \multirow{2}{*}{$\begin{array}{l}\text { Distance between } \\
\text { the plates }\end{array}$} & Capacitance(Farads) \\
\cline { 2 - 3 } & Theoretical & Simulated \\
\hline 250 & $3.04 \times 10^{-19}$ & $2.84 \times 10^{-19}$ \\
\hline 300 & $1.19 \times 10^{-18}$ & $1.08 \times 10^{-18}$ \\
\hline 350 & $4.64 \times 10^{-19}$ & $4.8 \times 10^{-19}$ \\
\hline 400 & $6.8 \times 10^{-19}$ & $6.98 \times 10^{-19}$ \\
\hline 450 & $6.19 \times 10^{-19}$ & $5.88 \times 10^{-19}$ \\
\hline 500 & $7.62 \times 10^{-19}$ & $6.79 \times 10^{-19}$ \\
\hline 550 & $6.28 \times 10^{-19}$ & $7.62 \times 10^{-19}$ \\
\hline 600 & $4.77 \times 10^{-19}$ & $4.9 \times 10^{-19}$ \\
\hline 650 & $8.47 \times 10^{-19}$ & $9 \times 10^{-19}$ \\
\hline
\end{tabular}

Variation of capacitance theoretically and in simulation with distance between the plates is shown in Fig 7.

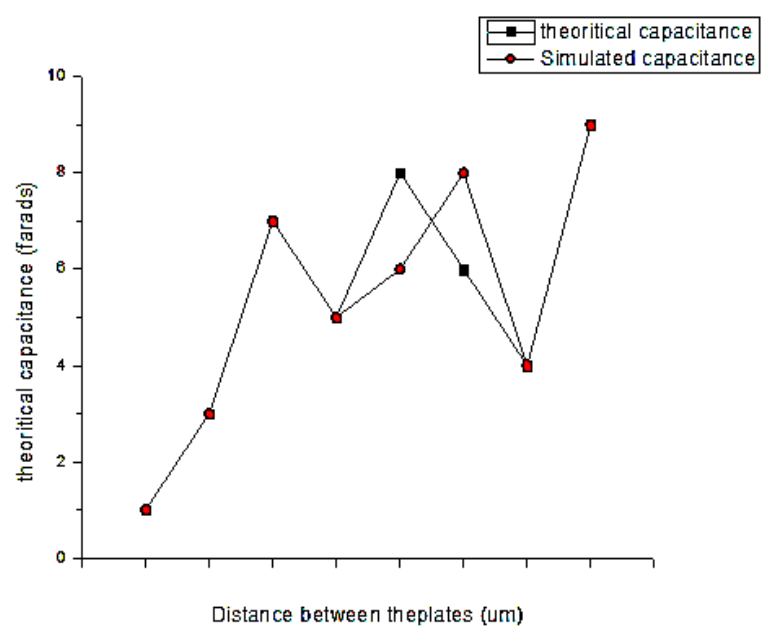

Fig. 7: Theoretical and Simulated Capacitance when the distance between the plates.

The capacitance is effected by the spacing between the plates which in-turn varies the output voltage of the proposed structure The output voltage so calculated for different values of spacing between the plate is given in table 4 .
Table 4: Output Voltage

\begin{tabular}{|c|c|}
\hline $\begin{array}{c}\text { Distance between the } \\
\text { plates }\end{array}$ & Voltage(Volts) \\
\hline 250 & $4.47 \times 10^{-9}$ \\
\hline 300 & $4.9 \times 10^{-9}$ \\
\hline 350 & $4.9 \times 10^{-9}$ \\
\hline 400 & $3.27 \times 10^{-9}$ \\
\hline 450 & $1.71 \times 10^{-9}$ \\
\hline 500 & $7.29 \times 10^{-9}$ \\
\hline 550 & $4.32 \times 10^{-9}$ \\
\hline 600 & $4.88 \times 10^{-9}$ \\
\hline 650 & $2.3 \times 10^{-9}$ \\
\hline
\end{tabular}

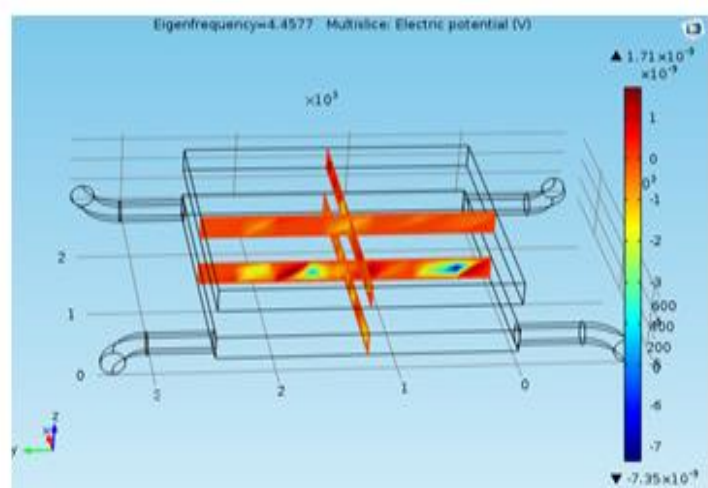

Fig. 8(a): Multi Slice Electrical Potential

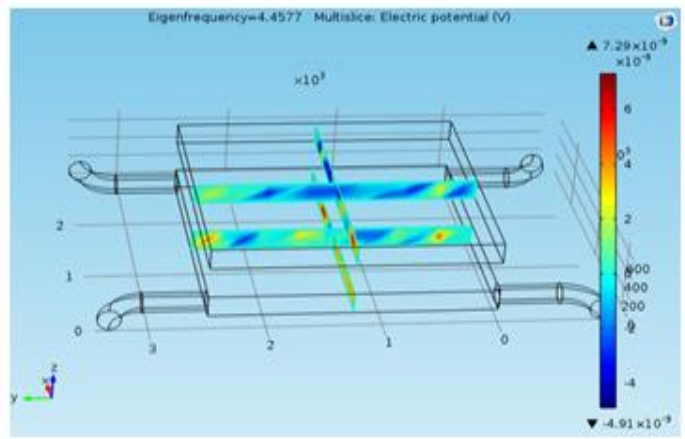

Fig. 8(b): Multi Slice Electrical Potentiale

The variation of output voltage with distance between the plates is shown in Fig 9

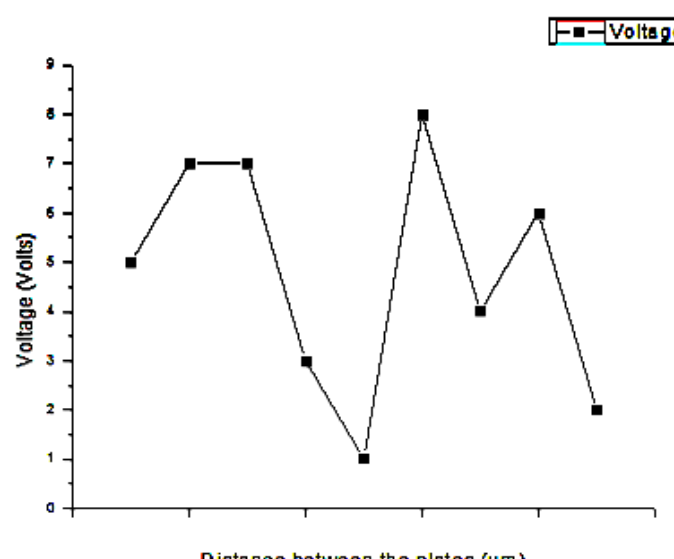

Fig. 9: variation of output voltage with distance between the plates 
An acceleration of $0.4 \mathrm{~g}$ to $0.8 \mathrm{~g}$ is applied on movable plate of the proof mass and the corresponding displacement values are noted and represented in table and shown in Figure 10 and table 5.

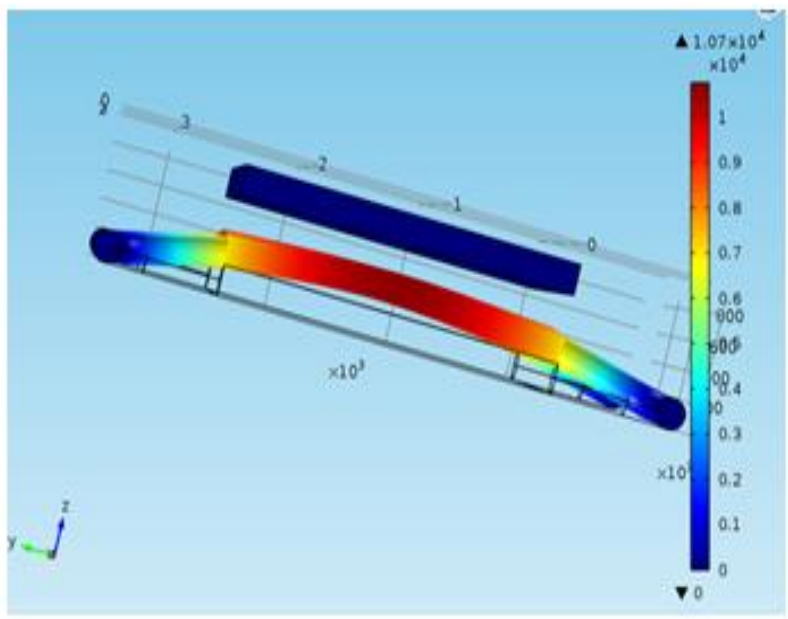

Fig. 10: Proposed model with applied acceleration

Table 5: Displacement for Applied Acceleration

\begin{tabular}{|c|c|}
\hline $\begin{array}{c}\text { Applied } \\
\text { Acceleration }\end{array}$ & Displacement $(\boldsymbol{\mu m})$ \\
\hline 0.4 & 5 \\
\hline 0.45 & 6 \\
\hline 0.5 & 6.7 \\
\hline 0.55 & 7.3 \\
\hline 0.6 & 8 \\
\hline 0.65 & 8.7 \\
\hline 0.7 & 9.4 \\
\hline 0.75 & 10.01 \\
\hline 0.8 & 10.07 \\
\hline
\end{tabular}

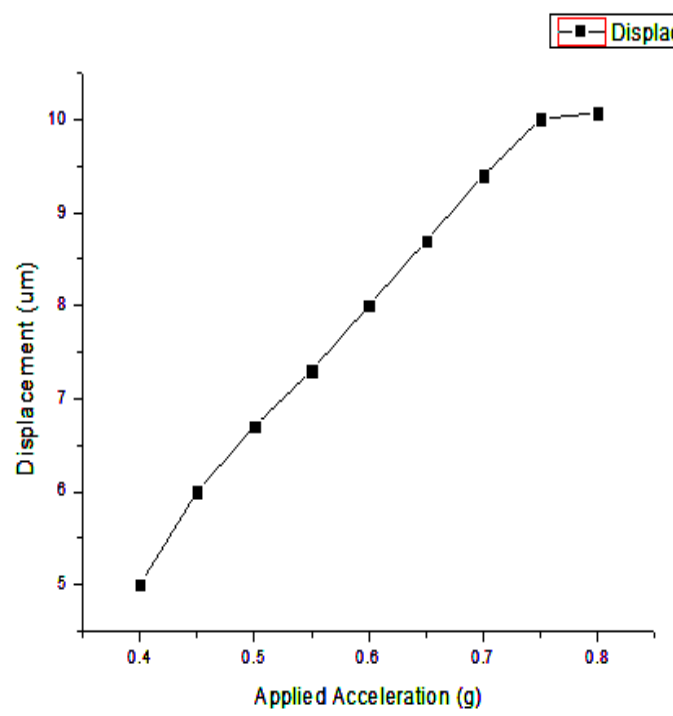

Fig. 11: variation of displacement with applied acceleration

From the fig 11 the displacement varies linearly with acceleration or force applied on the proof mass of the proposed sensor

An amplifier is designed with a gain of $103 \mathrm{db}$ and calculated the output voltage from the sensor. The value of output voltage is approximately 100 milli volts. The design and structure of the amplifier is shown in figure.

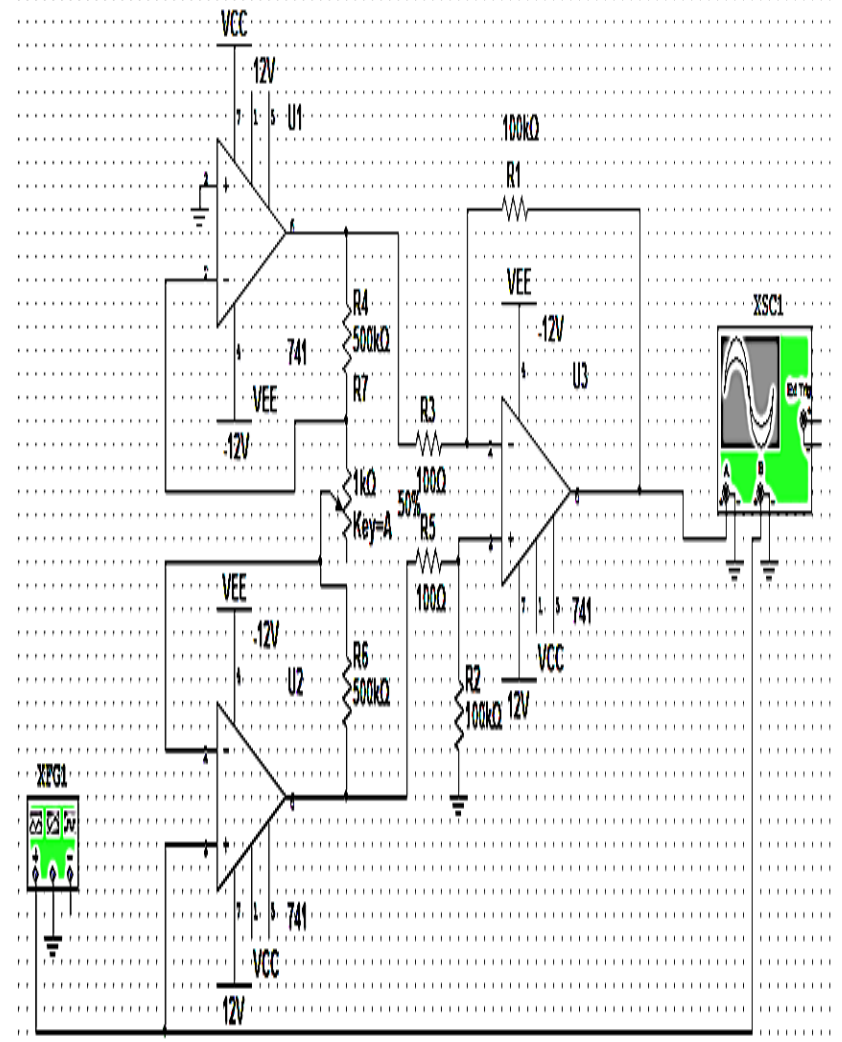

Fig. 12: Amplifier design with a gain of $103 \mathrm{db}$

The Frequency response curves of the designed amplifier are shown in figure 12.

For the case of amplifier both frequency Vs Phase and frequency Vs magnitude analysis was done and it is evident that the amplifier is in active region in a bandwidth of $1 \mathrm{~Hz}$ to $28 \mathrm{kHz}$.

\section{Conclusion}

In the proposed work we have followed a novel methodology for early detect the severity of Parkinson's Disease using MEMS sensors. The proposed device consists of two parallel plates in which one is stationary and another is fixed. When the acceleration of $0.4 \mathrm{~g}$ to $0.8 \mathrm{~g}$ is applied to the body of the proof mass and measured the variation on displacement, capacitance and voltage. We have designed an amplifier with a gain of $103 \mathrm{db}$ and measured the output voltage. Hence the proposed MEMS device gives accurate detection of Parkinson's disease. The displacement sensitivity, voltage sensitivity and capacitance sensitivity are calculated. The Proposed MEMS structure is attached to the finger tip of the patient and the tremor frequency is observed. If the tremor frequency is in the range of $3 \mathrm{~Hz}$ to $8 \mathrm{~Hz}$ then the decision is the patient is suffering with Parkinson's disease

\section{Acknowledgements}

The authors would like to thank NPMASS scheme for establishing national MEMS Design center in AP which is supported by IISC, Bangalore for providing the necessary design facilities.

\section{References}

[1] "MEMS and Microsystems: design , manufacture, and nanoscale engineering," 2nd Edition, by Tai-Ran Hsu, John Wiley \& Sons, Inc., Hoboken, New Jersey, 2008 (ISBN 978-0-470-08301-7) 
[2] Murthy K.S.N. et,al Design and simulation of MEMS bio sensor for the detection of Tuberculosis, International Journal of Science and Technology,2015,April,9(31):1-5.

[3] Jasti Sateesh et.al Design of MEMS bio sensor for Glucose Measurement, Journal of MEMS and Mechanics, 2015, April10(5):83-89.

[4] GRKPrasadet.al.AReviewonTechniquesfor Diagnosing and Monitoring Patients with Parkinson's Disease Journal of Biosensors \&Bioelectronics,2016, Vol:7(2).

[5] GRK Prasad et.al "Micro Electro Mechanical System Based Sensor For Early DetectionOf Parkinson's Disease - Design And Simulation",IntJ Pharm BioSci2017Jan; 8(1):(B)390-395.

[6] Hossein Pakdast,Triple coupled cantilever systems for mass detection and localization, Sensors and Actuators A: 2012 March 31,175: Pages 127-131

[7] N Siddaiah N, koti Reddy DR, Prasad GR, Pakdast H, BabuPS. optical and dielectric force gradient actuation schemes for excitation of triple coupled micro cantilever sensor in mass sensing applications, ARPN journalof Engineering and Applied Sciences,2015,May,10(8),3275-3279J. Clerk Maxwell, A Treatise on Electricity and Magnetism, 3rd ed., vol. 2. Oxford: Clarendon, 1892, pp.68-73

[8] Ramaker C, Marinus J, Stiggelbout AM, van Hilten BJ.Systematic evaluation of rating scales for impairment and disability in Parkinson's disease. Movement Disorders. 2002 Sep, 1;17(5):867-76.

[9] Patel S, Lorincz K, Hughes R, Huggins N,GrowdonJ,StandaertD,Akay M,Dy J,WelshM, Bonato P. Monitoring motor fluctuations in patients with Parkinson's disease using wearable sensors.IEEE Transactions On Information Technology In Biomedicine.2009Nov;13(6):864-73.

[10] Paul Pyzowski "Mems Applications For Treatment Of Nervous System Disorders" Mems Investor Journal

[11] PG Gopinath et.al "Microcantilever based Biosensor for Disease Detection Applications" Journal of Medical and Bioengineering Vol. 4, No. 4, August 2015

[12] Shyamal patel, et.al, " A review of sensors and systems with application in rehabilization" journal of neruro engineering and rehabilization, Vol.9, issue.21,P.No:1-17,2012.

[13] Athanasios Tsanas et.al "Accurate Telemonitoring of Parkinson's Disease Progression by Noninvasive Speech Tests", IEEE TRANSACTIONS ON BIOMEDICAL ENGINEERING, VOL. 57 NO. 4, APRIL 2010, P.No:884-893

[14] Ulrike Sommer et.al "Detection of Presymptomatic Parkinson's Disease: Combining 1 Smell Tests, Transcranial Sonography, and SPECT" 13 May 2004 in Wiley Inter Science, DOI: 10.1002/mds.20141, Vol. 19, No. 10, 2004, Pg.No:1-7.

[15] Ali Saad et.al, "Detection of freezing of gait for Parkinson's disease patients with multi-sensor device and Gaussian neural networks" Int. J. Mach. Learn. \& Cyber, DOI 10.1007/s13042-0150480-0, Pg.No:1-14.

[16] George Rigas et.al " Assessment of Tremor Activity in the Parkinson's Disease Using a Set of Wearable Sensors" IEEE TRANSACTIONS ON INFORMATION TECHNOLOGY IN BIOMEDICINE, VOL. 16, NO. 3, MAY 2012,Pg.No:478-487.

[17] Zack Zhu et.al "Real-Time Detection of Freezing of Gait for Parkinson's Disease Patients via Smartphone'P.No:1-4

[18] Sinziana Mazilu" Gait, Wrist, and Sensors: Detecting Freezing of Gait in Parkinson's Disease from Wrist Movement" Workshop on Sensing Systems and Applications Using Wrist Worn Smart Devices, 2015, 978-1-4799-8425-1/15/\$31.00 C2015 IEEE , P.No:583-588

[19] Benoit Mariani et.al, "On-Shoe Wearable Sensors for Gait and Turning Assessment of Patients With Parkinson's Disease" IEEE TRANSACTIONS ON BIOMEDICAL ENGINEERING, VOL. 60 , NO. 1, JANUARY 2013 Pg.No:155-158.

[20] K.Harish et.al "Tremor Quantification and its measurements on Parkinsonian patients", 978-1-4244-4764-0/09/\$25.00 (C2009 IEEE, Pg.No:1-3

[21] Jens Barth et.al, "Biometric and Mobile Gait Analysis for Early Diagnosis and Therapy Monitoring in Parkinson`s Disease " $33 \mathrm{rd}$ Annual International Conference of the IEEE EMBS Boston, Massachusetts USA, August 30 - September 3, 2011,Pg.No:868871.

[22] E. Raghuveer, K Hari Kishore, Shaik Shoukat Vali, and G. Siri Vennela "Verilog Implementation of UART with BIST Technique for TPG" International Journal of Pure and Applied Mathematics, ISSN No: 1314-3395, Vol No: 115, Issue No: 7, Page No: 531-536, September 2017
[23] Dr. Seetaiah Kilaru, Hari Kishore K, Sravani T, Anvesh Chowdary L, Balaji T "Review and Analysis of Promising Technologies with Respect to fifth Generation Networks", 2014 First International Conference on Networks \& Soft Computing, ISSN:978-1-47993486-7/14,pp.270-273,August2014.

[24] Meka Bharadwaj, Hari Kishore "Enhanced Launch-Off-Capture Testing Using BIST Designs" Journal of Engineering and Applied Sciences, ISSN No: 1816-949X, Vol No.12, Issue No.3, page: 636643, April 2017.

[25] N Bala Dastagiri, Kakarla Hari Kishore "Reduction of Kickback Noise in Latched Comparators for Cardiac IMDs" Indian Journal of Science and Technology, ISSN No: 0974-6846, Vol No.9, Issue No.43, Page: 1-6, November 2016.

[26] A Murali, K Hari Kishore, D Venkat Reddy "Integrating FPGAs with Trigger Circuitry Core System Insertions for Observability in Debugging Process" Journal of Engineering and Applied Sciences, ISSN No: 1816-949X, Vol No.11, Issue No.12, page: 2643-2650, December 2016

[27] Mahesh Mudavath, K Hari Kishore "Design of CMOS RF FrontEnd of Low Noise Amplifier for LTE System Applications Integrating FPGAs" Asian Journal of Information Technology, ISSN No: 1682-3915, Vol No.15, Issue No.20, page: 4040-4047, December 2016.

[28] P Bala Gopal, K Hari Kishore, B.Praveen Kittu "An FPGA Implementation of On Chip UART Testing with BIST Techniques", International Journal of Applied Engineering Research, ISSN 0973-4562, Volume 10, Number 14 , pp. 3404734051, August 2015

[29] S Nazeer Hussain, K Hari Kishore "Computational Optimization of Placement and Routing using Genetic Algorithm" Indian Journal of Science and Technology, ISSN No: 0974-6846, Vol No.9, Issue No.47, page: 1-4, December 2016.

[30] N Bala Gopal, K Hari Kishore "Analysis of Low Power Low Kickback Noise in Dynamic Comparators in Pacemakers" Indian Journal of Science and Technology, ISSN No: 0974-6846, Vol No.9, Issue No.44, page: 1-4, November 2016.

[31] M. Rajesh, Manikanthan, "ANNOYED REALM OUTLOOK TAXONOMY USING TWIN TRANSFER LEARNING”, International Journal of Pure and Applied Mathematics, ISSN NO:1314-3395, Vol-116, No. 21, Oct 2017.

[32] S.V.Manikanthan and T.Padmapriya "Recent Trends In M2m Communications In $4 \mathrm{~g}$ Networks And Evolution Towards 5g", International Journal of Pure and Applied Mathematics, ISSN NO:1314-3395, Vol-115, Issue -8, Sep 2017.

[33] T. Padmapriya and V. Saminadan, "Inter-cell Load Balancing Technique for Multi- class Traffic in MIMO - LTE - A Networks", International Conference on Advanced Computer Science and Information Technology, Singapore, vol.3, no.8, July 2015.

[34] S.V.Manikanthan and K.Baskaran "Low Cost VLSI Design Implementation of Sorting Network for ACSFD in Wireless Sensor Network", CiiT International Journal of Programmable Device Circuits and Systems,Print: ISSN 0974 - 973X \& Online: ISSN 0974 - 9624, Issue : November 2011, PDCS112011008. 